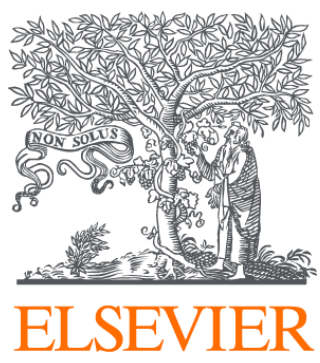

Since January 2020 Elsevier has created a COVID-19 resource centre with free information in English and Mandarin on the novel coronavirus COVID-

19. The COVID-19 resource centre is hosted on Elsevier Connect, the company's public news and information website.

Elsevier hereby grants permission to make all its COVID-19-related research that is available on the COVID-19 resource centre - including this research content - immediately available in PubMed Central and other publicly funded repositories, such as the WHO COVID database with rights for unrestricted research re-use and analyses in any form or by any means with acknowledgement of the original source. These permissions are granted for free by Elsevier for as long as the COVID-19 resource centre remains active. 


\title{
Health care visits during the COVID-19 pandemic: A spatial and temporal analysis of mobile device data
}

\author{
Jueyu Wang ${ }^{\text {a, }, \text { Noreen McDonald }}{ }^{\mathrm{a}}$, Abigail L. Cochran ${ }^{\mathrm{a}}$, Lindsay Oluyede ${ }^{\mathrm{a}}$, Mary Wolfe ${ }^{\mathrm{b}}$, \\ Lauren Prunkl ${ }^{\text {a }}$ \\ ${ }^{a}$ Department of City and Regional Planning, University of North Carolina at Chapel Hill, New East Building, CB3140, Chapel Hill, NC, 27599, United States \\ ${ }^{\mathrm{b}}$ Center for Health Equity Research, University of North Carolina at Chapel Hill, 323 MacNider Hall, 333 South Columbia Street, Chapel Hill, NC, 27599-7240, United \\ States
}

\section{A R T I C L E I N F O}

\section{Keywords:}

Healthcare access

Equity

COVID-19

Mobile device data

Time-series clustering

\begin{abstract}
A B S T R A C T
Transportation disruptions caused by COVID-19 have exacerbated difficulties in health care delivery and access, which may lead to changes in health care use. This study uses mobile device data from SafeGraph to explore the temporal patterns of visits to health care points of interest (POIs) during 2020 and examines how these patterns are associated with socio-demographic and spatial characteristics at the Census Block Group level in North Carolina. Specifically, using the K-medoid time-series clustering method, we identify three distinct types of temporal patterns of visits to health care facilities. Furthermore, by estimating multinomial logit models, we find that Census Block Groups with higher percentages of elderly persons, minorities, low-income individuals, and people without vehicle access are areas most at-risk for decreased health care access during the pandemic and exhibit lower health care access prior to the pandemic. The results suggest that the ability to conduct in-person medical visits during the pandemic has been unequally distributed, which highlights the importance of tailoring policy strategies for specific socio-demographic groups to ensure equitable health care access and delivery.
\end{abstract}

\section{Introduction}

Transportation is widely recognized as a critical factor in health care access (Syed et al., 2013). Nearly 5.8 million Americans in 2017 reported delaying medical care because of a lack of transportation options (Wolfe et al., 2020). COVID-19 has significantly disrupted transport and health systems. Early indications are that these changes, combined with lockdown requirements and a desire to limit exposure, reduced access to health care. For example, 35\% of US adults reported delaying health care because of COVID-19 (Household Pulse Survey, 2020, May), and many news outlets have reported decreases in preventive services (e.g., Smith, 2020; Martin et al., 2021).

In response to COVID-19, health care systems implemented policies that made accessing care both harder and easier. During the initial months of the pandemic, most systems eliminated or significantly reduced access to elective or non-emergency services. Hospitals also placed restrictions on whether patients could have individuals accompany them to appointments. While these policies reduced access to in- person health care, providers and insurers increased support for telemedicine visits (Chen et al., 2021; Medicare Telemedicine Health Care Provider Fact Sheet, 2020). COVID-19 also disrupted transportation options. Shared mobility options, including fixed-route transit, paratransit, and ride-hailing, became less available as agencies and firms decreased service in response to safety concerns and ridership declines (Hu and Chen, 2021; APTA, 2021).

The purpose of this study is to assess the impacts of COVID-19 on health care access in North Carolina (NC). We do this by using mobile device data from SafeGraph to identify visits to health care facilities and then use time-series clustering to identify Census Block Groups (CBGs) exhibiting similar medical visit patterns during 2020. We then examine the association between these temporal patterns and the sociodemographic and spatial characteristics of CBGs in NC. The findings reveal social and spatial inequalities in health care use before and during the COVID-19 pandemic. As part of our work, we also assess the reliability of the mobile device data.

The research contributes to existing studies on the impacts of the

\footnotetext{
* Corresponding author.

E-mail addresses: Oliva.wang@unc.edu (J. Wang), noreen@unc.edu (N. McDonald), acochran@unc.edu (A.L. Cochran), oluyede@live.unc.edu (L. Oluyede), mary_wolfe@med.unc.edu (M. Wolfe), lauren.prunkl@unc.edu (L. Prunkl).
} 
pandemic on activity-travel behavior in two aspects. First, mobile phone data provides a viable data source to capture large-scaled human mobility and has been widely used to map mobility patterns and identify activity hotspots during the COVID-19 pandemic. However, few studies (e.g., Atkinson et al., 2020) have checked the quality of mobile phone data to measure mobility during the COVID-19 pandemic. As a result, it is difficult to know the validity of these findings. Our study validates the use of mobile phone data to measure mobility patterns and activities by using weekly pattern data from SafeGraph, specifically for health care related activities. Second, the research examines the correlation between socio-demographic and spatial characteristics and medical visits during the pandemic. A better understanding of these associations could inform the design of appropriate policies to deliver health care services in a safe, equitable, and timely manner.

\section{Background}

\subsection{Health care services during COVID-19}

The COVID-19 pandemic drastically changed peoples' access to and use of health care services. These changes include health insurance loss, health facility closure, and the increasing use of telemedicine (Chen et al., 2021). During the pandemic, more than 40 million Americans lost their jobs, which further caused many of them to lose their employer-based health insurance (Blumenthal et al., 2020). As a result, they may have been forced to delay necessary but noncritical treatments (Blumenthal et al., 2020). These impacts also tend to be disproportionately distributed. Minorities and people with low educational attainment and low incomes have experienced disproportionate job loss and delayed care (Kurtzleben, 2020).

The pandemic also caused significant economic threats to the viability of some health care providers, especially those located in rural and poor communities (Blumenthal et al., 2020; Chen et al., 2021). The pre-existing accessibility to health care facilities in rural and poor communities is comparatively lower (Ghorbanzadeh et al., 2021; Guida and Carpentieri, 2021). Many providers were temporarily closed during the pandemic, which may have further decreased geographic access to local health care and further influenced health care use.

Telemedicine is another key change in health care services. Many health care systems increased telemedicine options for patients during the pandemic. Health insurers also expanded their coverage to include telemedicine. With these changes, the use of telemedicine increased significantly during the pandemic to replace in-person care (Mann et al., 2020). However, not everyone has equal access to telemedicine. People with limited internet access, language barriers, and cognitive limitations may not be able to use telemedicine, and telemedicine is not suitable for all medical services (Chen et al., 2021).

\subsection{Transportation during COVID-19}

Transportation provides access to health care. Despite the increasing popularity of telemedicine, transportation is still important for people who need in-person care. Thus, the pandemic's disruptions of travel demand and transportation services may influence health care access and use. In response to stay-at-home orders and social distancing regulations, travel demand decreased (Dasgupta et al., 2020; Pepe et al., 2020). Some people may also have reduced their health care trips, delayed medical visits, or used telemedicine to meet critical health care needs (Cochran, 2020). Others may have had more time and flexibility to commit to conducting health care activities because of their flexible work schedules. Mobility reduction also varies among different socio-demographic groups and geographic locations (Dasgupta, 2020; Pepe et al., 2020; McLaren 2020). Thus, the ability to conduct medical trips may also vary among people with different socio-demographic characteristics and living in different geographic locations. Neighborhoods with higher percentages of minorities and people with low incomes and low educational attainment tended to show less reduction in mobility perhaps due to reduced options for remote work (Dasgupta, 2020; Pepe et al., 2020; McLaren 2020). Jones (2020) documented that $54 \%$ of urban residents, $42 \%$ of suburban residents, and $27 \%$ of rural residents viewed COVID-19 as a major threat. The disparities in COVID-19 threat awareness may partially contribute to the geographic differences in mobility change. Scholsser et al. (2020) revealed that mobility declined more in large cities in Germany compared to less dense population areas. Do Lee et al. (2021) found that population density is positively associated with more mobility reduction in the United States.

Transportation services also changed. Nationally, public transit ridership dropped by $80 \%$ at the start of the pandemic and remained approximately 60\% below 2019 levels (APTA, 2021). Public transit agencies cut services because of reduced ridership and revenues. For example, in response to reduced revenues, Los Angeles' transit agency (LA Metro) cut its budget by 1.2 billion and service by $20 \%$ (Nelson, 2020). MARTA, in the Atlanta region, cut most of its bus routes in April and is still operating at low capacity, and King County Metro in Seattle cut service by $15 \%$ in September (Bellis, 2020). Ride-sourcing programs, such as Uber and Lyft, suspended their pooled and shared ride options in response to the spread of COVID-19. Changes in public transit and ride-hailing programs left some people, particularly those who rely more on transit and shared rides, such as individuals with disabilities, facing greater challenges accessing transportation and health care (Cochran, 2020).

\subsection{Mobile phone data and measuring mobility}

Mobile phone data, which consist of Call Detail Records (CDR) or Global Position System (GPS) data, have been widely used in transportation research. These data offer a rich source of information on continuous space-time geography in urban areas. These data have been used to develop human mobility models (e.g., Deville et al., 2016), to develop traffic models (e.g., Demissie et al., 2018; Breyer et al., 2018), and to estimate trip rates (e.g., Çolak et al., 2015).

Given the popularity of mobile device data in mobility studies, its representativeness has attracted increasing attention. Ranjan et al. (2012) assessed the accuracy of CDR data in measuring human mobility. They revealed that sparsely sampled CDRs have biases, which are associated with the ratio of CDRs in an individual's trajectory. GPS data comparatively have fine granularity in spatial and temporal aspects. However, because people's phone activities in space and time are uneven, mobile phone location data also suffers from the problem of sparse sampling (Becker et al., 2013). As a result, mobile device data based on GPS locations also introduces biases in measuring human mobility. For example, Lu et al. (2017) analyzed the representativeness of mobile phone location data on the estimation of human mobility. They revealed that mobile phone location data underestimates human mobility as mobile phone location data is incomplete.

Because mobile phone data can capture large-scale human mobility patterns, it also has been used in COVID-19 related studies to map human mobility patterns (e.g., Gao et al., 2020), identify activity hotspots (e.g., Li et al., 2021), and set parameters for disease transmission models (e.g., Chang et al., 2021). Chen et al. (2021) and Kang et al. (2020) demonstrated that the aggregate trends derived from SafeGraph data match the aggregate trends revealed in Google Mobility Data in the US.

\section{Study area and data}

\subsection{Study area}

Our study area is North Carolina (NC), consisting of three large metropolitan areas, Charlotte Metro, Research Triangle Area, and Piedmont Triad. In response to COVID-19, NC declared a state of 
emergency on March 10, 2020, and issued stay-at-home orders on March 14,2020 , to close all K-12 public schools and ban gatherings of more than 100 people. Additional stay-at-home orders were implemented continually over March to close non-essential businesses and enforce social distancing measures. On May 8, 2020, NC moved to phase 1, reopening retail businesses and childcare facilities. On May 22, 2020, NC moved to phase 2, reopening restaurant dine-in services; bars and nightclubs with capacity limits; and allowing gatherings of 10 people. On September 1, 2020, NC moved to phase 2.5, reopening indoor exercise facilities and increasing mass gathering limits to 25 people indoors and 50 people outdoors. On October 2, 2020, NC moved to phase 3 , allowing the reopening of bars, entertainment venues, and movie theaters with capacity restrictions. On December 8, 2020, NC further lifted stay-at-home orders but continued encouraging people to stay home between 10 p.m. and 5 a.m.; and required restaurants, bars, entertainment venues, personal care businesses, and other businesses to close at 10:00 p.m.

\subsection{Data}

We obtained data on visits to medical facilities in NC from SafeGraph, a data company that aggregates anonymized location data from mobile device applications. SafeGraph data is a type of GPS data, tracking devices which opted in via apps with GPS. It tracks the movement of mobile devices from their home CBGs to points of interest (POIs) across the United States. SafeGraph defines each device's home CBG as the most common nighttime location over the previous six weeks. The study period spans 52 weeks from January 6, 2020, to the week starting on December 28, 2020.

Specifically, we used SafeGraph's Core Places and Weekly Patterns datasets to identify trips to health care facilities. For each POI, these datasets provide the North American Industry Classification System (NAICS) code as well as estimates of weekly visits and visitors and the home CBG of each visitor. Weekly visits are the aggregated raw counts of visits with the duration at least $4 \mathrm{~min}$ to the POI per week, and weekly visitors are the aggregated number of unique devices to the POI per week.

We aggregated estimates of weekly visitors from each home CBG to all medical POIs. Medical facilities are POIs with the designation "office of physicians (NAICS code 621,111)", "office of dentists (NAICS code 621,210 ), "office of other health practitioners (NAICS code 6213), "office of outpatient care centers" (NAICS code 6214), and "general medical and surgical hospitals" (NAICS code 622,110) (Table S-1 in Supplementary Materials). Medical facility POIs are usually concentrated spatially, especially in urban areas. It is challenging to measure visitors to each medical POI accurately. Furthermore, multiple medical POIs are usually identified for a large medical facility. For example, POIs of the office of physicians are inaccurately identified within the building boundary of the Duke University Hospital. These POI data issues further challenge the accuracy of assigning visits to each medical POI and differentiating visits to different types of medical facilities. Thus, for each CBG, we aggregated the estimates of weekly visitors to all these types of health care facilities.

We used American Community Survey (ACS) 2019 5-Year Estimates to measure socio-demographic and economic characteristics at the CBG level. We included metrics in five domains: (1) age; (2) race and ethnicity; (3) education; (4) economic status; and (5) transportation disadvantage. We also derived the percent of the population without internet access and the percent of commuters working at home as proxy measures for the potential of using telemedicine from ACS 2019 5-Year Estimates.

For spatial variables, we used the urban-rural classification scheme from the National Center for Health Statistics to categorize CBGs into six types: large central metropolitan, large fringe metropolitan, medium metropolitan, small metropolitan, micropolitan, or noncore county. We also calculated population density for each $\mathrm{CBG}$, defined as the number of people per square mile. We further used the medical POI data from SafeGraph to derive the measure of the density of health care facilities, defined as the count of the number of health care facilities per square mile for each CBG.

\section{Methods}

Our study aimed to assess the reliability of SafeGraph data for analyzing trips to medical facilities and patterns of travel to medical facilities during 2020 .

\subsection{Reliability of SafeGraph data}

To assess the reliability of SafeGraph data, we used three approaches. First, we estimated sample geographic representativeness by comparing the number of sampled devices with 2019 Census Bureau population counts at different geographic levels, from the CBG, Census Tract, county, and state levels. Census Tracts are designed to be relatively homogeneous units in terms of population characteristics, economic status, and living conditions and have a population of 4000 (U.S. Census Bureau Definition, 2021). A CBG is a subdivision of a Census Tract and is a geographic unit that typically has a population of 600 and 3000 people. The CBG is also the smallest geographic entity for which the sample data from the decennial census is available. Using American Community Survey 2019 5-year estimates, we also estimated the expected demographic characteristics of sampled devices and compared to state averages. Second, we compared SafeGraph medical facility POIs with the Centers for Medicare and Medicaid Services (CMS) list of health care providers (CMS, 2020) to check the accuracy and representativeness of medical facility POIs. Third, we compared and correlated SafeGraph estimates of medical facility visit volumes with the in-person outpatient visit volume to facilities under the UNC Health Care system. The in-person encounter visit volume data was obtained from Carolina Data Warehouse for Health.

\subsection{Analysis of temporal travel trends to medical facilities}

\subsubsection{Data preprocessing}

We conducted several preprocessing steps on the medical visitor flow data to ensure that CBGs contained sufficient and valid records to derive stable estimates of visitors for analyzing temporal patterns. We removed CBGs with zero population, as sampled devices in CBGs with zero population are likely to be misidentified. The number of devices in some CBGs dropped significantly across 2020 from thousands or hundreds to only a few. To address this, we included CBGs where weekly counts of sampled devices were at least $2 \%$ of the CBG's population and removed CBGs with fewer than 10 sampling devices. The analysis results are not sensitive to our selection of cut points. Our final preprocessing yielded 52 weeks of data for 5565 of the 6155 NC CBGs. The number of devices sampled for each CBG in the SafeGraph data varies each week. Because of this, we normalized aggregated number of visitors from each CBG to medical facility POIs by the reported number of sampled devices in the CBG and focused our analysis on the number of medical visitors per device per week from each CBG.

\subsubsection{Time-series clustering}

After preprocessing, each CBG has a time series sequence with a length of 52, representing the number of medical care visitors per device per week across 52 weeks in 2020. We have 5655 (CBGs) time series sequences. We employed time-series clustering to group CBGs with similar temporal patterns in medical care visitors per device together. Time-series clustering partitions time series datasets into clusters based on a similarity measure (Das et al., 1998; Aghabozorgi et al., 2015).

We experimented with two common distance measures for determining the similarity, Euclidean distance and Dynamic Time Warpping (DTW) distance. Euclidean distance is a common measure of similarity 
in clustering analysis Keogh and Pazzani (1999); 2001. However, Euclidean distance for time-series datasets requires the exact alignment of the time axis and is very sensitive to small distortion in the time axis (Keogh and Pazzani, 1999; 2001) (See Equation (1)); Euclidean distance requires that the ith point in one sequence is exactly aligned with the ith point in the other. Thus, we chose a distance measure based on dynamic time warpping, with a window size of 2 for warpping, which allows us to compare the similarity in the absolute number of medical visitors per device in time and therefore allows for small distortions of the time axis (See Figure S-1). Equation (2) represents the DTW distance between any two time-series sequences.

$\operatorname{Dist}($ Euclidean $)=\sqrt{\sum_{i=0}^{n}\left(p_{i}-q_{i}\right)^{2}}$

where $\mathrm{p}$ and $\mathrm{q}$ are two time-series sequences of length $\mathrm{n}(\mathrm{n}=52$ here); $\mathrm{i}$ is time index, representing the week number.

$\operatorname{Dist}(D T W)=\min \sqrt{\sum_{k=1}^{k}\left(w_{k}\right)}$

To determine the DTW distance for $\mathrm{p}$ and $\mathrm{q}$, we firstly derive a $\mathrm{n}$ by $\mathrm{n}$ distance matrix between $\mathrm{p}$ and $\mathrm{q}, \mathrm{D}$. The value of individual cell $\left(\mathrm{d}_{\mathrm{ij}}\right)$ in the matrix D is calculated as $\sqrt{\left(p_{i}-q_{j}\right)^{2}} \cdot w_{k}$ is the cell $(i, j)_{k}$ in matrix D that is also the $k^{\text {th }}$ element of a wrapping path, $W=\left\{w_{1}, w_{2}, \ldots w_{k}\right\}$. A wrapping path is a series of neighboring elements in the distance matrix, $\mathrm{D}$ that links the bottom left cell $\left(w_{1}=d_{11}\right)$ with the top right cell $\left(w_{k}=\right.$ $\left.d_{n n}\right)$. There would be many wrapping paths from the bottom left cell to the top right cell. We are interested in the wrapping path with the minimized length. DTW uses the following dynamic programming to find the shortest path (See Equation (3) (4)).

$D^{p, q}(i, j)=\sqrt{\left(p_{i}-q_{j}\right)^{2}}+\min \left(D^{p, q}(i-1, j-1), D^{p, q}(i-1, j), D^{p, q}(i, j-1)\right)$

$|i-j| \leq \Delta t$

where $D^{p, q}(i, j)$ is the sum of current cell $\left(\mathrm{d}_{\mathrm{ij}}\right)$ and the minimum of the cumulative distances of the adjacent cells. The resulting $D^{p, q}(i, j)$ denotes the DTW distance between p and q. $\Delta t$ is the wrapping window. It is a constraint on the wrapping path searching. Euclidean distance has a wrapping window size of 0 . Unconstrainted DTW has a wrapping window size of n-1. In the study, we choose a wrapping window size of 2 .

Various clustering algorithms are available (Aghabozorgi et al., 2015; Jain, 2010). K-means and K-medoid are commonly used. K-means is often used in conjunction with the Euclidean distance, and K-medoid is more appropriate for time-series clustering with DTW (Aghabozorgi et al., 2015). Thus, we adopted the K-medoid clustering algorithm. The core steps of k-medoid clustering are as following: (1) specify the number of clusters $k$; (2) select $k$ samples from time-series objects as the initial center of the k clusters; (3) assign each object to the nearest center based on the DTW distance; (4) find the center within each cluster, the object with the minimum average DTW distance to the remaining objects; and (5) repeat steps (3) and (4) until none of the objects change their cluster memberships.

In this study, we present results with three clusters. We selected $\mathrm{k}=3$ by running the DTW distance-based K-Medoid clustering algorithm with values of $\mathrm{k}$ from 2 to 7 . The clustering outcomes of different numbers of clusters were visually compared and explored (see Supplementary Materials for details). We selected the value of $k=3$ based on our exploration.

\subsubsection{Statistical analysis}

We characterized differences across time-series clusters by comparing socioeconomic characteristics using unadjusted (ANOVA) and adjusted (multinomial logit regression) approaches.

\section{Results}

\subsection{Reliability of SafeGraph medical facilities data}

The SafeGraph sample averaged 631,835 devices in NC during 2020. The proportion of population sampled (sampled device counts/state population) ranged from 4.5 to $8 \% 2020$ (Fig. 1). The number of sampling devices decreased significantly during the lockdown period (from Mid-March to May). SafeGraph sources data from phone applications, such as navigation and social media apps, where people could opt into location tracking. Thus, stay-at-home orders may decrease the use of apps with location tracking and therefore decrease the number of sampled devices. At the county level, the sample averaged 6318 devices (6\% of population; IQR 5\%-7\%). At the census tract level, the sample averaged 287 devices ( $6 \%$ of total population; IQR 5\%-7\%). At the CBG level, the sample averaged 102 devices ( $6 \%$ of total population; IQR 4\%$8 \%$ ). The correlation coefficients between average device counts and Census population estimates ranged from 0.98 to 0.99 at the county level, 0.77 to 0.85 at the census tract level, and 0.72 to 0.83 at the CBG level. The ratio of devices to census population also varied spatially with the ratio being higher in metropolitan areas compared to nonmetropolitan areas (Fig. 2).

We analyzed how closely the device data matched state demographic averages by assuming that sampled devices in a CBG have the same demographic characteristics as the CBG (Table 1). In terms of age composition, the weekly device sample across 2020 is slightly overrepresented for the over age 45 population while under-represented for those under age 45 . But overall, the device sample is well-sampled across age groups. The sample is generally over-represented on Whites but under-represented on non-Whites (those identifying as Black and Hispanic). The sample is generally well-sampled for educational attainment categories. It is slightly over-represented for those with higher education levels and under-represented for those with high school degrees or below. The sample is under-represented for lower household income categories (annual income between $\$ 15,000$ and $\$ 35,000$, and less than $\$ 15,000$ ) and over-represented for higher household income categories (income between $\$ 50,000$ and $\$ 100,000$, and higher than $\$ 100,000)$.

Compared with the CMS list of medical facilities, the spatial distribution of medical POIs from SafeGraph is well-balanced. The distribution of medical facilities at the county level derived from both datasets is similar; counties with a higher number of CMS providers also have a higher number of medical facility POI from the SafeGraph dataset (Pearson correlation coefficient $=0.89$ ). There are no medical facilities in 594 census tracts (27\%), based on the two datasets. 42 tracts have one or two CMS providers, which are not identified in the SafeGraph POI. There are no medical facilities in 3316 CBGs (54\%), based on both datasets. 107 CBGs have one or two CMS providers, which are not identified in SafeGraph POI. The correlation coefficients at the census tract and CBG levels are moderate, at 0.58 and 0.54 , respectively. As mentioned before, medical POIs are usually concentrated spatially, and multiple POI points are often identified for large medical facilities. For example, POIs of offices of physicians in SafeGraph are often identified within the boundary of medical centers. Thus, one provider in the CMS list often corresponds to several POIs in SafeGraph POI data. The data issues may contribute to the moderate correlation coefficients ( 0.58 at the tract level and 0.54 at the CBG level) at the tract and CBG levels.

Overall, trends of medical facility visits from SafeGraph and the UNC Health Care system are comparable, exhibiting similar temporal patterns (Fig. 3). The number of medical visits started to drop in the middle of March when NC's governor declared a State of Emergency and reached their lowest values in early April. Visits to facilities in the UNC Health Care system dropped more (over 70\%) during the lockdown period than visits measured from SafeGraph data. The gaps may result 


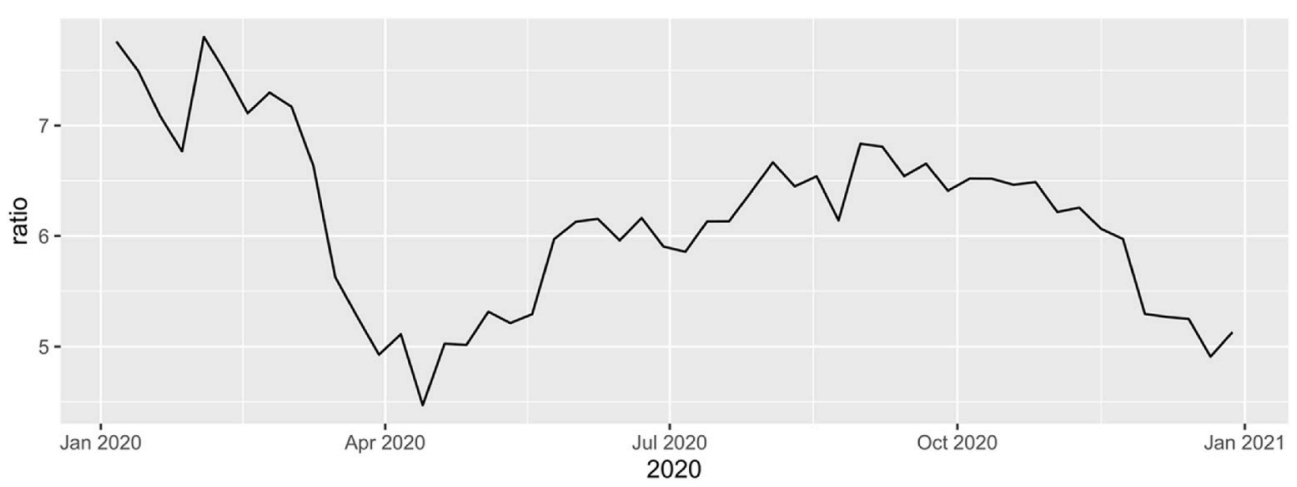

Fig. 1. Weekly sampled device counts vs. state population from ACS 2015-2019.

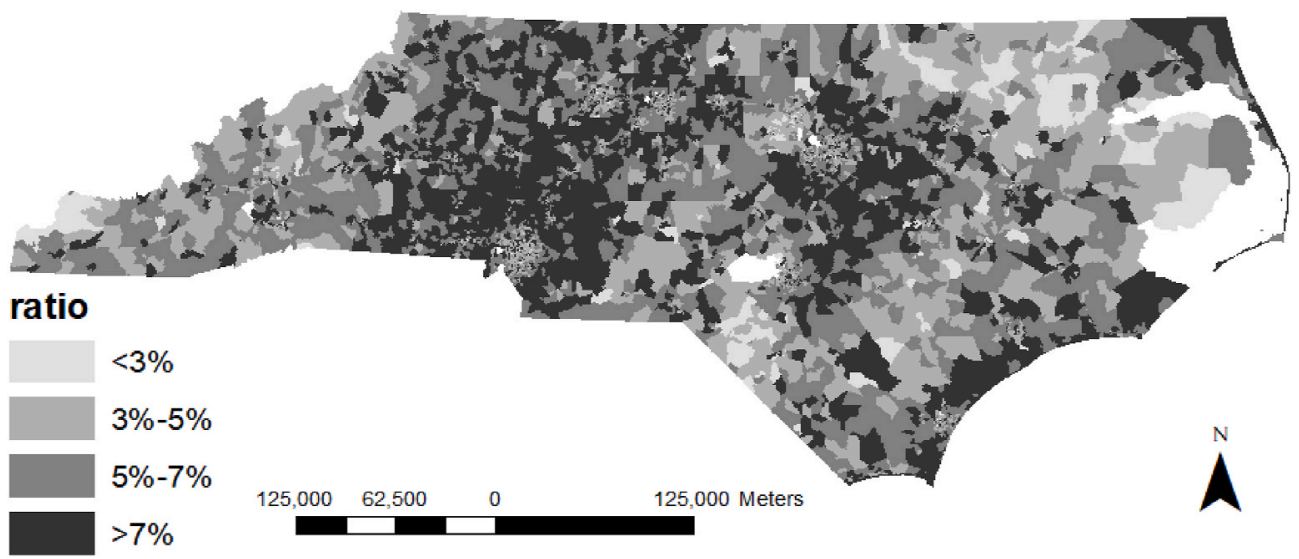

Fig. 2. Ratio of mean weekly sampled device counts in 2020 to the population from the ACS 2019 estimates at CBG level. NA represents zero population.

Table 1

Comparison of device demographics to Census demographics for NC.

\begin{tabular}{|c|c|c|c|}
\hline & $\begin{array}{l}\text { Sample Mean (range } \\
\text { across } 52 \text { weeks) }\end{array}$ & $\begin{array}{l}\text { Census } \\
\text { Bureau }\end{array}$ & $\begin{array}{l}\text { Ratio (sample/ } \\
\text { census) }\end{array}$ \\
\hline$\%$ Female & $51.3(51.2-51.4)$ & 51.3 & $1.00(1.00-1.00)$ \\
\hline \multicolumn{4}{|l|}{ Age groups } \\
\hline \% Under 18 & $22.1(21.9-22.4)$ & 22.4 & $0.99(0.98-1.00)$ \\
\hline$\% 18-44$ & $34.5(33.9-35.3)$ & 35.5 & $0.97(0.95-0.97)$ \\
\hline$\% 45-65$ & $27.0(26.6-27.3)$ & 26.3 & $1.03(1.01-1.04)$ \\
\hline \% Over 65 & $16.2(15.8-16.6)$ & 15.9 & $1.02(1.00-1.04)$ \\
\hline \multicolumn{4}{|l|}{ Race and ethnicity } \\
\hline$\%$ White & $66.6(65.1-67.8)$ & 63.1 & $1.06(1.03-1.07)$ \\
\hline$\%$ Black & $18.7(17.9-19.8)$ & 21.1 & $0.89(0.85-0.94)$ \\
\hline \% Hispanic & $8.6(8.4-8.9)$ & 9.4 & $0.92(0.89-0.95)$ \\
\hline \multicolumn{4}{|l|}{ Education } \\
\hline $\begin{array}{l}\% \text { High school } \\
\text { or below }\end{array}$ & $37.2(36.7-37.7)$ & 37.9 & $0.98(0.97-1.00)$ \\
\hline$\% \mathrm{BA}$ or more & $31.5(30.8-32.2)$ & 31.3 & $1.01(0.99-1.03)$ \\
\hline \multicolumn{4}{|c|}{ Household income (\$) } \\
\hline $\begin{array}{l}\text { \% Less than } 15 \\
\mathrm{k}\end{array}$ & $10.9(10.6-11.1)$ & 11.4 & $0.95(0.93-0.97)$ \\
\hline$\% 15 \mathrm{k}-35 \mathrm{k}$ & $20.1(19.9-20.3)$ & 20.6 & $0.98(0.97-0.99)$ \\
\hline$\% 35 \mathrm{k}-50 \mathrm{k}$ & $13.7(13.6-13.8)$ & 13.9 & $0.98(0.98-0.99)$ \\
\hline$\% 50 \mathrm{k}-100 \mathrm{k}$ & 30.7 (30.5-30.9) & 30.4 & $1.01(1.00-1.01)$ \\
\hline$\% 100 \mathrm{k}+$ & $24.7(24.0-25.1)$ & 23.7 & $1.04(1.03-1.06)$ \\
\hline
\end{tabular}

Note: Calculation of sample demographics assumes sampled devices in a CBG have the same demographic characteristics as the CBG.

from the disparities in what types of visits were recorded in the two datasets. SafeGraph captures all visits to health care facilities in NC, including inpatient visits, outpatient visits, and all other types of visits such as those by employees. The in-person encounter volume to clinics in the UNC Health Care system data only contains outpatient care visits.
Compared to inpatient and employee visits, outpatient visits were more likely to be deferred during the lockdown. Furthermore, most medical facilities in the UNC Health Care system are in central urban areas where declines in travel during the lockdown were greater than in more outlying and rural areas (Do Lee et al., 2021; Schlosser et al., 2020) (Figure S-2). In May, when the stay-at-home order was lifted and NC entered Phase 2, medical visits recovered, as revealed by both datasets. The correlation coefficient between the two groups is strong, at 0.83 .

Based on these assessments, we concluded that, overall, SafeGraph data are well-balanced in terms of geographic and demographic representativeness of population but slightly under-represented in minority and low-income groups and have a well-balanced sampling of POIs. The aggregated trends in medical visits revealed in SafeGraph data somewhat matched up to the aggregated trends inpatient volumes to facilities in the UNC Health Care system. However, we still need to be cautious that SafeGraph data have limited coverage in outlying and rural areas; limited representativeness in low-income populations who often have limited access to smartphones with GPS; limited accuracy of POI location identification; and limited coverage for POIs of small size. As a result, medical visits derived from $\mathrm{SafeGraph}$ by low-income people, and medical visits to small size hospital POIs, especially in rural areas, may not be accurately recorded in SafeGraph data.

\subsection{Temporal patterns of medical facility visits}

We identified three clusters of CBGs that exhibited similar temporal trends for trips to medical facilities. Medical care visits of CBGs in all three clusters dropped at the start of the pandemic and did not return to pre-pandemic levels by the end of 2020 (Fig. 4). 


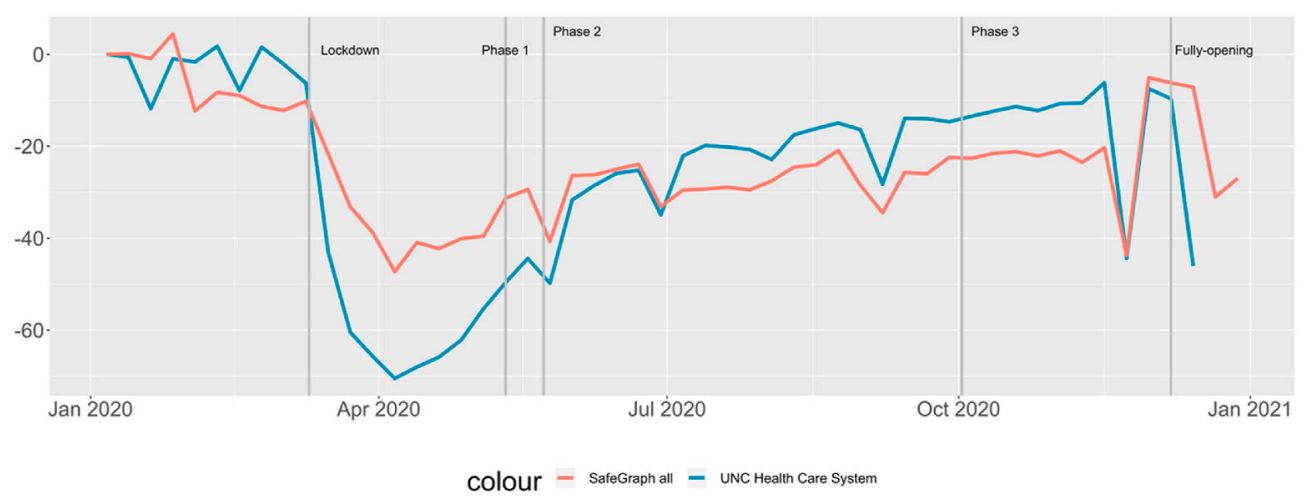

Fig. 3. Temporal trends of medical facility visit change.

Note: Visits to facilities under UNC health Care system are available until the week starting on July 12,

2020. Two lines represent the percent change in visits (UNC Health Care system) and visits per device (SafeGraph) since the first week of 2020.

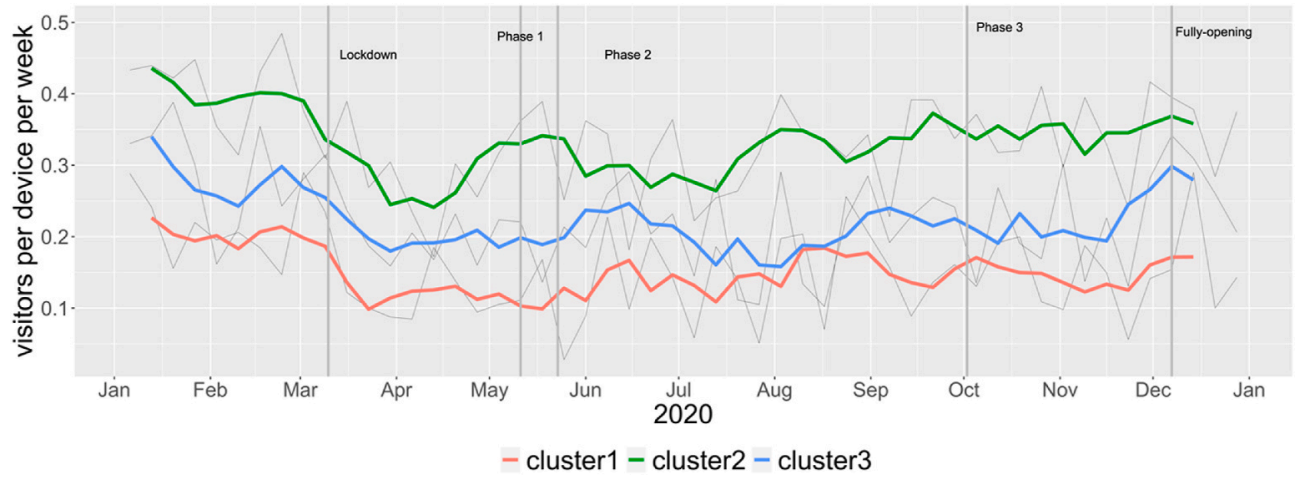

Fig. 4. Clustering Results (Smoothed Lines) of DTW K-Medoid Clustering Algorithms: Three Identified Clusters.

Note: grey lines represent the medoids of the three identified clusters.

- Cluster 1. CBGs in this cluster $(\mathrm{n}=1899)$ have lower medical visits all the time. These CBGs also responded to the lockdown in April with a strong reduction in medical visits. As the COVID-19 restrictions were gradually lifted, these CBGs experienced a moderate and slow increase in medical visits.

- Cluster 2. CBGs in this cluster $(\mathrm{n}=1208)$ have higher numbers of medical visits per device per week and responded to the stay-at-home orders implemented in March and April strongly; CBGs in this cluster saw a significant decrease in medical visits during the lockdown period. Compared to CBGs in cluster 1, medical visits bounced back sooner but were lower than pre-pandemic levels when NC implemented a re-opening phase in mid-May.
- Cluster 3. Compared to CBGs in cluster 2, CBGs in this cluster ( $\mathrm{n}=$ 2458) have a medium level of medical visits. CBGs in this cluster also experienced a moderate decrease in medical visits during the lockdown period and a moderate and low increase after.

\subsection{Spatial distribution of clusters}

Fig. 5 shows the spatial distribution of the three clusters across NC. CBGs in cluster 1 are more likely to be in central urban core and outlying rural areas. CBGs in cluster 2 are more concentrated in the suburban areas of large metropolitan areas. CBGs in cluster 3 are more spatially dispersed. The spatial patterns imply that socio-demographic and spatial

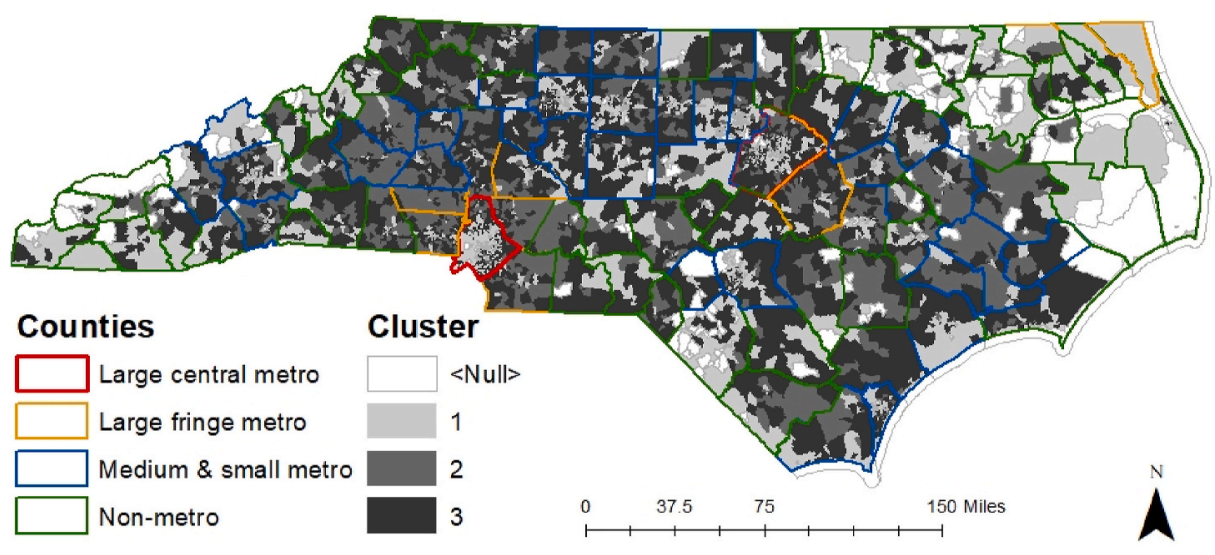

Fig. 5. Spatial distribution of the three clusters. 
variables may explain observed disparities in medical care-seeking before and during the pandemic.

\subsection{Descriptive analysis}

CBGs in the three clusters are significantly different on all selected socio-demographic variables (Table 2). CBGs in cluster 2 comparatively have a higher percentage of persons over age 65 . For racial composition, CBGs in clusters 2 and 3 have higher percentages of Whites. CBGs in clusters 1 and 3 have higher percentages of Blacks and Hispanics. As for education, compared to CBGs in cluster 1, CBGs in cluster 2 and 3 have high percentages of adults with higher educational attainment. In terms of household income, CBGs in cluster 2 and 3 show a similar distribution of a lower percentage of low-income households and a higher percentage of high-income households, while CBGs in cluster 2 have a comparatively higher percentage of households with annual incomes greater than $\$ 100,000$. Compared to CBGs in cluster 2 and 3, CBGs in cluster 1 have a higher percentage of zero-car households and households without internet access.

Significant disparities in all spatial variables (except non-metro variables) are revealed among CBGs in the three clusters. $20 \%$ of CBGs in cluster 1 are in large central areas, and $48 \%$ of CBGs in cluster 1 are in medium and small metros. $21 \%$ of CBGs in cluster 2 are in fringe areas of large metros. In periphery non-metro areas (micropolitan and noncore), the distribution of CBGs in the three clusters is similar. CBGs in cluster 1 and 3 tend to have a higher population density.

\subsection{Regression results}

We estimated a multinomial logit model to examine how sociodemographic and spatial characteristics at the CBG level are associated with cluster types (Table 3; Table 4). We chose cluster 2 as the reference cluster because CBGs in cluster 2 comparatively have higher medical visits, and CBGs in cluster 2 exhibit a typical pattern of medical visits, decreasing significantly during the lockdown and recovering after. The socio-demographic variables are strongly correlated, and thus we presented our final model without any multicollinearity issues. The model overall has a moderate fit (Pseudo $\mathrm{R}^{2}=0.10$ ).

CBGs in cluster 1- the cluster with the lowest rate of medical visits and slowest recovery in the rate of visits - have a higher proportion of residents over age 65, with incomes under 35,000, without household vehicles, and with higher pre-pandemic rates of telework. These CBGs also have a lower proportion of residents that self-identify as White.

CBGs in cluster 3, compared to those in cluster 2-the cluster with slower recovery in the rate of in-person visits during the re-opening stages, have a lower proportion of residents with incomes over $\$ 100,000$ and who identify as White.

Spatial variables play an important role in determining temporal patterns of visits to medical POIs. CBGs in cluster 1 are more likely to be in large central metros and less likely to be located in large fringe metros. These CBGs also tend to have higher population density and less geographic proximity to health care POIs. Compared to CBGs in Cluster 2 , CBGs in cluster 3 are less likely to be located in large fringe metros and are generally located in areas with fewer number of health care POIs.

\section{Discussion}

Our study aimed to analyze patterns of travel to medical facilities during 2020 and assess the reliability of SafeGraph data for analyzing trips to medical facilities.

\subsection{Disparate patterns of visits to medical facilities}

We found three distinct clusters of temporal patterns of visits to medical POI during 2020. All three clusters experienced a reduction in medical care visits during the lockdown but differed in their extent and recovery patterns.

CBGs with lower medical visits before the pandemic (cluster 1) experienced a slower recovery. CBGs with higher percentages of elderly persons, minorities, low-income individuals, and people without vehicle access (cluster 1) had limited use of health care before and during the pandemic and experienced a slower recovery after the lockdown. These socio-demographic disparities confirm the necessity of health systems to care adequately for these groups under normal conditions and during a pandemic.

CBGs with higher population density and in central areas are more likely to be in cluster 1 . Higher population density areas and central areas are usually areas with higher public transit use (Taylor and Fink, 2003), but public transit is also highly likely to be affected by the pandemic. As a result, people living in these areas may be more likely to have difficulty accessing health care during COVID-19 and experience a lower recovery after the lockdown. The positive association between a

Table 2

Socio-demographics characteristics by the three clusters.

\begin{tabular}{|c|c|c|c|c|c|c|c|c|}
\hline & \multicolumn{2}{|c|}{ Cluster 1} & \multicolumn{2}{|c|}{ Cluster 2} & \multicolumn{2}{|c|}{ Cluster 3} & \multicolumn{2}{|l|}{ ANOVA } \\
\hline & mean & std & mean & std & mean & std & $\mathrm{P}$-value & Sig \\
\hline & \multicolumn{8}{|c|}{ Socio-demographics } \\
\hline$\%$ Age over 65 & 17.1 & 10.6 & 18.5 & 7.8 & 17.9 & 8.8 & 0.000 & $* * *$ \\
\hline$\%$ White & 54.1 & 29.9 & 77.0 & 19.1 & 68.8 & 23.1 & 0.000 & $* * *$ \\
\hline$\%$ Black & 29.3 & 26.6 & 12.4 & 15.4 & 17.6 & 18.6 & 0.000 & $* * *$ \\
\hline$\%$ Hispanic & 10.7 & 12.2 & 6.2 & 8.0 & 8.2 & 9.5 & 0.000 & $* * *$ \\
\hline \% Below high school & 42.3 & 18.3 & 38.9 & 16.7 & 39.2 & 17.4 & 0.000 & $* * *$ \\
\hline$\%$ Bachelor and above & 26.7 & 19.7 & 29.0 & 19.7 & 29.2 & 19.6 & 0.000 & $* * *$ \\
\hline$\%$ Income $<35 \mathrm{k}$ & 40.5 & 18.9 & 29.6 & 14.4 & 31.2 & 15.7 & 0.000 & $* * *$ \\
\hline$\%$ Income $35-50 \mathrm{k}$ & 14.9 & 8.6 & 13.3 & 7.3 & 14.2 & 7.8 & 0.000 & $* * *$ \\
\hline$\%$ Income $50 \mathrm{k}-100 \mathrm{k}$ & 27.9 & 12.0 & 31.2 & 10.3 & 31.2 & 10.3 & 0.000 & $* * *$ \\
\hline$\%$ Income $100 \mathrm{k}+$ & 16.7 & 15.1 & 25.9 & 17.3 & 23.4 & 17.2 & 0.000 & $* * *$ \\
\hline$\%$ No vehicle households & 9.1 & 10.3 & 4.2 & 5.1 & 4.8 & 5.9 & 0.000 & $* * *$ \\
\hline$\%$ No internet access & 19.8 & 14.0 & 16.0 & 11.5 & 16.2 & 11.8 & 0.000 & $* * *$ \\
\hline \multirow[t]{2}{*}{$\%$ Work from home } & 5.0 & 5.9 & 5.0 & 5.4 & 5.4 & 5.5 & 0.065 & \\
\hline & \multicolumn{8}{|c|}{ Spatial Characteristics } \\
\hline Large central metro & 20.1 & & 9.6 & & 16.7 & & 0.000 & $* * *$ \\
\hline Large fringe metro & 6.8 & & 21.1 & & 14.8 & & 0.000 & $* * *$ \\
\hline Medium \& small metro & 47.5 & & 43.7 & & 42.8 & & 0.007 & $* *$ \\
\hline Non-metro & 25.6 & & 25.6 & & 25.7 & & 0.994 & \\
\hline Population density (1000 residents/sq.miles) & 1.7 & 1.9 & 0.8 & 1.1 & 1.1 & 1.5 & 0.000 & $* * *$ \\
\hline Health care facility density & 4.1 & 11.7 & 4.5 & 25.6 & 3.3 & 12.0 & 0.076 & \\
\hline
\end{tabular}

Note: $* * *$ significance at $\mathrm{p}<0.001 ; * *$ significance at $\mathrm{p}<0.01 ; *$ significance at $\mathrm{p}<0.05$. 
Table 3

Modeling results.

\begin{tabular}{|c|c|c|c|c|c|c|c|c|}
\hline & \multicolumn{4}{|c|}{ Cluster 1 (vs. Cluster 2) } & \multicolumn{4}{|c|}{ Cluster 3 (vs. Cluster 2) } \\
\hline & Coef. & SE & Sig. & & Coef. & SE & Sig. & \\
\hline & \multicolumn{8}{|c|}{ Socio-demographics } \\
\hline$\%$ Age over 65 & 0.020 & 0.005 & 0.000 & $* * *$ & 0.010 & 0.005 & 0.034 & * \\
\hline$\%$ White & -0.025 & 0.002 & 0.000 & $* * *$ & -0.014 & 0.002 & 0.000 & $* * *$ \\
\hline$\%$ Income $<35 \mathrm{k}$ & 0.013 & 0.004 & 0.001 & $* * *$ & -0.004 & 0.004 & 0.340 & \\
\hline$\%$ Income $100 \mathrm{k}+$ & -0.026 & 0.004 & 0.000 & $* * *$ & -0.017 & 0.003 & 0.000 & $* * *$ \\
\hline$\%$ No vehicle households & 0.024 & 0.008 & 0.002 & $* *$ & -0.003 & 0.008 & 0.707 & \\
\hline \multirow[t]{2}{*}{$\%$ Work from home } & 0.050 & 0.009 & 0.000 & $* * *$ & 0.030 & 0.008 & 0.000 & $* * *$ \\
\hline & \multicolumn{8}{|c|}{ Spatial Characteristics } \\
\hline \multicolumn{9}{|c|}{ Spatial locations (ref. $=$ non-metro) } \\
\hline Large central metro & 0.766 & 0.184 & 0.000 & $* * *$ & 0.502 & 0.168 & 0.003 & $* *$ \\
\hline Large fringe metro & -0.894 & 0.144 & 0.000 & $* * *$ & -0.300 & 0.115 & 0.009 & $* *$ \\
\hline Medium-small metro & 0.055 & 0.103 & 0.590 & & -0.052 & 0.093 & 0.576 & \\
\hline Pop density & 0.297 & 0.042 & 0.000 & $* * *$ & 0.159 & 0.040 & 0.000 & $* * *$ \\
\hline Health care facility density & -0.012 & 0.003 & 0.000 & $* * *$ & -0.010 & 0.003 & 0.000 & $* * *$ \\
\hline Constant & 1.159 & 0.270 & 0.000 & $* * *$ & 1.851 & 0.250 & 0.000 & $* * *$ \\
\hline Log Likelihood & -5326 & & & & & & & \\
\hline Pseudo R2 & 0.100 & & & & & & & \\
\hline
\end{tabular}

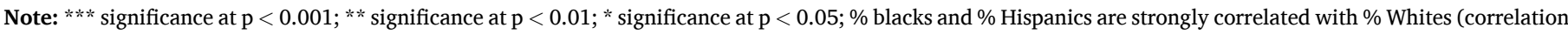

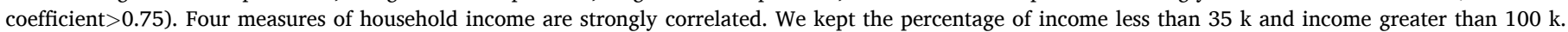

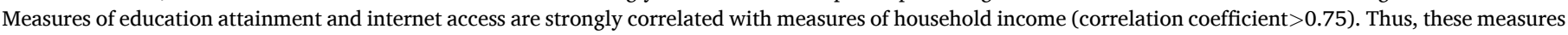
were removed from the final model.

Table 4

Elasticities.

\begin{tabular}{|c|c|c|c|}
\hline & Cluster 1 & Cluster 2 & Cluster 3 \\
\hline & \multicolumn{3}{|c|}{ Socio-demographics } \\
\hline$\%$ Age $65+$ & $0.16^{* * *}$ & $-0.19 * * *$ & -0.02 \\
\hline$\%$ White & $-0.72^{* * *}$ & $0.89 * * *$ & -0.05 \\
\hline$\%$ Income less than $35 \mathrm{k}$ & $0.31 * * *$ & -0.13 & $-0.25^{* * *}$ \\
\hline$\%$ Income $100 \mathrm{k}+$ & $-0.25^{* * *}$ & $0.32^{* * *}$ & -0.03 \\
\hline$\%$ No vehicle households & $0.08^{* * *}$ & -0.07 & $-0.09 * * *$ \\
\hline \multirow[t]{2}{*}{$\%$ Work from home } & $0.10^{* * *}$ & $-0.16^{* * *}$ & 0.003 \\
\hline & \multicolumn{3}{|c|}{ Spatial variables } \\
\hline \multicolumn{4}{|c|}{ Spatial locations (ref. = non-metro) } \\
\hline Large central metro & $0.28 * * *$ & $-0.48^{* * *}$ & 0.02 \\
\hline Large fringe metro & -0.46 & $0.44 * * *$ & $0.14^{*}$ \\
\hline Medium-small metro & 0.06 & 0.004 & -0.05 \\
\hline Pop density & $0.12^{* * *}$ & $-0.25^{* * *}$ & $-0.05^{* *}$ \\
\hline Health care facility density & $-0.02 *$ & $0.03^{* * *}$ & -0.006 \\
\hline
\end{tabular}

Note: $* * *$ significance at $\mathrm{p}<0.001 ; * *$ significance at $\mathrm{p}<0.01 ; *$ significance at $\mathrm{p}<0.05$.

lower facility density and the likelihood of being in cluster 1 also suggests the limited physical access to health care resources for CBGs in cluster 1, highlighting the importance of ensuring equal access to health care resources (Guida and Carpenteri, 2021).

CBGs in the other two clusters with comparably higher medical visits (cluster 2 and cluster 3); cluster 3 has a relatively slower and modest recovery. The socio-demographic and spatial characteristic variables also explain the disparities in recovery patterns between cluster 2 and cluster 3 . It is important to note that these significant associations tend to be small in magnitude, suggesting recovery patterns of medical care visits after the lockdown are not sensitive to socio-demographic and spatial characteristics. However, the effect sizes of two variables, percentages of White individuals and the highest-income individuals are still comparable. CBGs with higher percentages of Whites and people in the highest income class (greater than $\$ 100,000$ ) have the highest medical visits and reduced their medical visits during the lockdown but increased their visits soon after the lockdown.

Taken together, these results suggest that areas most at-risk for decreased health care access during a pandemic are the same neighborhoods where residents exhibited lower health care access prior to the pandemic.

\subsection{Using mobile phone data to measure medical trips}

Our assessment of the accuracy and reliability of mobile device data from SafeGraph to analyze visits to medical facilities shows that the data has good geographic representativeness of population at different geographic scales (county, tract, and $\mathrm{CBG}$ ). The data was spatially balanced when sampling health care facility POIs and could measure the overall temporal patterns of visits to medical facility POIs at the state level. However, the study limits the comparison between outpatient visits to clinics in the UNC Health Care system and medical care visits of SafeGraph, which calls for more research on assessing the accuracy in using mobile device data on measuring visits to POIs. Furthermore, the data still suffers from a slight under-representativeness of low-income people and non-White individuals. The data issues may indicate that SafeGraph data does not accurately record medical visits by low-income people, especially in rural areas. The study's focus on longitudinal analysis and spatially balanced distribution of health care POI would help alleviate potential sampling bias.

Our assessment also showed a significant decrease in sampled devices, which may be attributed to the inability of mobile phone data to track a population staying at home not using phone apps with GPS tracking. However, SafeGraph data does not reveal the information about which phone apps with GPS tracking are recorded in the data collection process. Different socio-demographic groups may use their phone apps differently. Without data transparency about phone apps, it would be difficult for us to evaluate and correct the potential bias of the sampling.

\subsection{Strengths and limitations}

Timely delivery and access to health care are essential under normal circumstances and during a pandemic. This study is among the few to examine medical care visits during COVID-19. Distinct from other studies, ours is based on a geographically extensive sample of mobile devices across the state of NC, allowing for interpretation beyond the context of a single geographic setting and a small sample of patients.

Several caveats should be considered when interpreting the research results. First, the data suffers from some under-representativeness issues revealed in our assessment. The data tracks a device instead of an individual, and thus it could not distinguish multiple people traveling with one sampled device. These data issues are still unclear, and this study does not differ from most studies using mobile device data. 
Second, the data also cannot capture sub-CBG without individual information. This study focuses on area-level associations rather than individual-level associations; however, area-level characteristics are significantly associated with health behavior independent of individual characteristics (Turrell et al., 2010), suggesting the value of considering area-level characteristics. Third, this study only examined the data of year 2020 and may conflate the seasonality with the impacts of COVID-19, which calls for future studies with the incorporation of data of previous years. Finally, we only considered the state-wide restrictions and did not consider the disparities in restrictions across counties. Counties may have had different levels of restrictions during COVID-19, and future studies could benefit from considering more fine-scale restriction disparities.

While our focus on NC was useful for understanding broader geographic disparities, especially urban and rural disparities, it could also mask variation within metropolitan areas which usually have more apparent disparities in the distribution of socio-demographic characteristics. Thus, it would be interesting to conduct similar research in a single metropolitan area as a supplement to this study's findings.

\section{Conclusion and policy implications}

Analysis of the temporal patterns of visits to medical POI across 2020 and their associations with socio-demographic and spatial characteristics at the CBG level reveals two key findings. The findings may be useful for policymakers seeking to improve health care delivery and access.

$C B G s$ with higher percentages of elderly persons, minorities, low-income individuals, and people without vehicle access (cluster 1) had lower use of health care before the pandemic and experienced a slower recovery in medical visits after the lockdown. Health policymakers and transportation planners need to develop appropriate strategies to address persistent inequalities in health care use by these social groups. First, health policymakers need to make telemedicine a viable option for people living in these less-advantaged CBGs. Historically vulnerable populations, such as racial minorities, adults over age 65 , and low-income households, have limited digital literacy and access (Smith, 2020). Community health centers, which provide safety-net care for low-income and uninsured people, also have financial constraints to implement telemedicine (Kim et al., 2020). Health care providers need to develop training programs to teach populations in these areas the digital skills to use telemedicine and offer language interpreter access. Health care systems may also need to provide community health centers located in less-advantaged areas with funding to support telemedicine.

Second, public transportation agencies, private transportation providers, health care providers, and governments should work together to provide low-cost and reliable transportation options for people living in these less advantaged CBGs. Transportation agencies should ensure that transit and paratransit options for health care are still operating for these CBGs. People may also be less willing to use transit to conduct health care visits because of safety concerns. Thus, transportation and health care agencies may need to make efforts to partner with private transportation providers, like ride-hailing services (e.g., Uber), to provide low-cost ride-hailing options for vulnerable populations to access care.

CBGs in the central areas of large metropolitans or with higher population density tend to have a slow recovery of health care visits (cluster 1 and $3 v$ s. cluster 2). The results may be attributed to significant disruptions in transportation in these areas. When transit services become unavailable in these dense and central areas, transportation agencies, health care providers, and private transportation providers should support alternate transportation options for people living in central and dense areas. Ridehailing companies like Uber and Lyft have provided Non-Emergency Medical Transportation (NEMT) since 2018 in select geographies. Studies have shown that using ridesharing NEMT has produced positive results, such as fewer missed appointments (Power et al., 2016). Health care providers and insurers could continue partnerships with ride-hailing companies for people living in these areas to access health care. Transportation agencies could also collaborate with bike-share companies to provide free or low-cost bike-share in these areas. Bike share programs in Chicago, Boston, and New York have offered health care workers free access during the pandemic (BicycleRetailer, 2021). Bicycling may not be a good transportation option for all people who need care. However, the availability of bike-share programs could expand mode options and reduce the number of transit transfers for people who live in the central areas and rely on transit to access health care.

\section{Acknowledgements}

This work was supported by the North Carolina Policy Collaboratory with funding from the North Carolina Coronavirus Relief Fund established and appropriated by the North Carolina General Assembly and a contract from the Southeastern Transportation Research, Innovation, Development and Education Center (STRIDE), a Regional University Transportation Center sponsored by a grant from the US Department of Transportation's University Transportation Center Program.

\section{Appendix A. Supplementary data}

Supplementary data to this article can be found online at https://doi. org/10.1016/j.healthplace.2021.102679.

\section{References}

Aghabozorgi, S., Shirkhorshidi, A.S., Wah, T.Y., 2015. Time-series clustering-a decade review. Inf. Syst. 53, 16-38.

American Public Transit Association (APTA), 2021. The Impact of the COVID-19 Pandemic on Public Transit Funding Needs in the US. Retrieved from. https://www. apta.com/wp-content/uploads/APTA-COVID-19-Funding-Impact-2021-01-27.pdf.

Atkinson, T., Dolmas, J., Koch, C., Koenig, E.F., Mertens, K., Murphy, A., Yi, K.M., 2020. Mobility and Engagement Following the SARS-Cov-2 Outbreak. Federal Reserve Bank of Dallas, Research Department.

Dec Bellis, R., 2020. What services cut are transit agencies facing around the country? Transportation for America Blog, Retrieved from. https://t4america.org/2020/12 /01/what-service-cuts-are-transit-agencies-facing-around-the-country/.

Becker, R., Cáceres, R., Hanson, K., Isaacman, S., Loh, J.M., Martonosi, M., et al., 2013. Human mobility characterization from cellular network data. Commun. ACM 56 (1), 74-82.

Breyer, N., Gundlegård, D., Rydergren, C., 2018. Cellpath routing and route traffic flow estimation based on cellular network data. J. Urban Technol. 25 (2), 85-104.

Blumenthal, D., Fowler, E.J., Abrams, M., Collins, S.R., 2020. Covid-19-implications for the health care system. N. Engl. J. Med. 383, 1483-1488. https://doi.org/10.1056/ NEJMsb2021088.

Chang, S., Pierson, E., Koh, P.W., Gerardin, J., Redbird, B., Grusky, D., Leskovec, J., 2021a. Mobility network models of COVID-19 explain inequities and inform reopening. Nature 589 (7840), 82-87.

Chen, K.L., Brozen, M., Rollman, J.E., Ward, T., Norris, K.C., Gregory, K.D. Zimmerman, F.J., 2021. How is the COVID-19 pandemic shaping transportation access to health care? Transportation Research Interdisciplinary Perspectives 100338 .

Cms, 2020. https://www.cms.gov/Research-Statistics-Data-and-Systems/Downloadabl e-Public-Use-Files/Provider-of-Services.

Cochran, A.L., 2020. Impacts of COVID-19 on access to transportation for people with disabilities. Transportation Research Interdisciplinary Perspectives 8, 100263.

Çolak, S., Alexander, L.P., Alvim, B.G., Mehndiratta, S.R., González, M.C., 2015. Analyzing cell phone location data for urban travel: current methods, limitations, and opportunities. Transport. Res. Rec. 2526 (1), 126-135.

August Das, G., Lin, K.I., Mannila, H., Renganathan, G., Smyth, P., 1998. Rule discovery from time series. KDD 98 (1), 16-22.

Dasgupta, N., Jonsson Funk, M., Lazard, A., White, B.E., Marshall, S.W., 2020. Quantifying the Social Distancing Privilege Gap: a Longitudinal Study of Smartphone Movement. Available at: SSRN 3588585.

Demissie, M.G., Phithakkitnukoon, S., Kattan, L., 2018. Trip distribution modeling using mobile phone data: emphasis on intra-zonal trips. IEEE Trans. Intell. Transport. Syst. 20 (7), 2605-2617.

Deville, P., Song, C., Eagle, N., Blondel, V.D., Barabási, A.L., Wang, D., 2016. Scaling identity connects human mobility and social interactions. Proc. Natl. Acad. Sci. Unit. States Am. 113 (26), 7047-7052.

Do Lee, W., Qian, M., Schwanen, T., 2021. The association between socioeconomic status and mobility reductions in the early stage of England's COVID-19 epidemic. Health Place 102563.

Gao, S., Rao, J., Kang, Y., Liang, Y., Kruse, J., 2020. Mapping county-level mobility pattern changes in the United States in response to COVID-19. SIGSpatial Special 12 (1), 16-26. 
Ghorbanzadeh, M., Kim, K., Ozguven, E.E., Horner, M.W., 2021. Spatial accessibility assessment of COVID-19 patients to healthcare facilities: a case study of Florida. Travel Behaviour and Society 24, 95-101.

Guida, C., Carpentieri, G., 2021. Quality of life in the urban environment and primary health services for the elderly during the Covid-19 pandemic: an application to the city of Milan (Italy). Cities 110, 103038.

May Household Pulse Survey, 2020. United States Census Bureau.

$\mathrm{Hu}, \mathrm{S}$., Chen, P., 2021. Who left riding transit? Examining socioeconomic disparities in the impact of COVID-19 on ridership. Transport. Res. Transport Environ. 90, 102654.

Jain, A.K., 2010. Data clustering: 50 years beyond K-means. Pattern Recogn. Lett. 31 (8), 651-666.

March Jones, 2020. Urban Residents in States Hit Hard by COVID-19 Most Likely to See it as a Threat to Daily Life. Pew Research Center. retrieved from. https://www.pew research.org/fact-tank/2020/03/20/urban-residents-in-states-hit-hard-by-covid-19most-likely-to-see-it-as-a-threat-to-daily-life/.

Kang, Y., Gao, S., Liang, Y., Li, M., Rao, J., Kruse, J., 2020. Multiscale dynamic human mobility flow dataset in the US during the COVID-19 epidemic. Scientific data 7 (1), $1-13$.

September Keogh, E.J., Pazzani, M.J., 1999. Scaling up dynamic time warping to massive datasets. In: European Conference on Principles of Data Mining and Knowledge Discovery. Springer, Berlin, Heidelberg, pp. 1-11.

Keogh, E.J., Pazzani, M.J., 2001. Derivative dynamic time warping. In: Proceedings of the 2001 SIAM International Conference on Data Mining, vol. 370. SIAM, pp. 1-11.

June Kim, J.-H., Desai, E., Cole, M.B., 2020. How the rapid shift to telehealth leaves many community health centers behind during the COVID-19 pandemic. Health Affairs Blog. Retrieved from. https://www.healthaffairs.org/do/10.1377/hblog20 200529.449762/full/.

Kurtzleben, D., 2020. Job Losses Higher Among People of Color during Coronavirus Pandemic. Retrieved from. https://www.npr.org/2020/04/22/840276956/minoriti es-often-work-these-jobs-they-were-among-first-to-go-in-coronavirus-layo.

Li, Q., Bessell, L., Xiao, X., Fan, C., Gao, X., Mostafavi, A., 2021. Disparate patterns of movements and visits to points of interest located in urban hotspots across US metropolitan cities during COVID-19. Royal Society open science 8 (1).

Lu, S., Fang, Z., Zhang, X., Shaw, S.L., Yin, L., Zhao, Z., Yang, X., 2017. Understanding the representativeness of mobile phone location data in characterizing human mobility indicators. ISPRS Int. J. Geo-Inf. 6 (1), 7.

Mann, D.M., Chen, J., Chunara, R., Testa, P.A., Nov, O., 2020. COVID-19 transforms health care through telemedicine: evidence from the field. J. Am. Med. Inf. Assoc. 27 (7), 1132-1135.
Martin, K., Kuroswki, D., Given, P., Kennedy, K., Clayton, E., 2021. The Impact of COVID-19 on the Use of Preventive Health Care. Health Care Cost Institute. Retrieved from. https://healthcostinstitute.org/hcci-research/the-impact-of-covid19-on-the-use-of-preventive-health-care.

McLaren, John, 2020. Racial Disparity in COVID-19 Deaths: Seeking Economic Roots with Census Data. http://www.nber.org/papers/w27407.

May Medicare Telemedicine Health Care Provider Fact Sheet, 2020. CMS.Gov, retrieved from: https://www.cms.gov/newsroom/fact-sheets/medicare-telemedicine-healthcare-provider-fact-sheet.

Sept Nelson, L., 2020. L.A. Metro cuts budgets by 1.2 billion, slashed services by $20 \%$, Los Angeles Times retrieved from. https://ktla.com/news/local-news/l-a-metro-cuts -budget-by-1-2-billion-slashes-services-by-20/.

Pepe, E., Bajardi, P., Gauvin, L., Privitera, F., Lake, B., Cattuto, C., Tizzoni, M., 2020. COVID-19 outbreak response, a dataset to assess mobility changes in Italy following national lockdown. Scientific data 7 (1), 1-7.

Powers, B.W., Rinefort, S., Jain, S.H., 2016. Nonemergency medical transportation: delivering care in the era of Lyft and Uber. J. Am. Med. Assoc. 316 (9), 921-922. https://doi.org/10.1001/jama.2016.9970.

Ranjan, G., Zang, H., Zhang, Z.-L., Bolot, J., 2012. Are call detail records biased for sampling human mobility? ACM SIGMOB - Mob. Comput. Commun. Rev. 16 (3), 33. https://doi.org/10.1145/2412096.2412101.

Schlosser, F., Maier, B.F., Jack, O., Hinrichs, D., Zachariae, A., Brockmann, D., 2020. COVID-19 lockdown induces disease-mitigating structural changes in mobility networks. Proc. Natl. Acad. Sci. Unit. States Am. 117 (52), 32883-32890.

October Smith, T., 2020. Preventive Care: as Pandemic Stretches on, "No More Time to Wait". AMA. retrieved from. https://www.ama-assn.org/delivering-care/patient-s upport-advocacy/preventive-care-pandemic-stretches-no-more-time-wait.

Syed, S.T., Gerber, B.S., Sharp, L.K., 2013. Traveling towards disease: transportation barriers to health care access. J. Community Health 38 (5), 976-993.

Taylor, B.D., Fink, C.N., 2003. The Factors Influencing Transit Ridership: A Review and Analysis of the Ridership Literature.

Turrell, G., Haynes, M., Burton, N.W., Giles-Corti, B., Oldenburg, B., Wilson, L.A., et al., 2010. Neighborhood disadvantage and physical activity: baseline results from the HABITAT multilevel longitudinal study. Ann. Epidemiol. 20 (3), 171-181.

Wolfe, M.K., McDonald, N.C., Holmes, G.M., 2020. Transportation barriers to health care in the United States: findings from the national health interview survey, 1997-2017. Am. J. Publ. Health 110 (6), 815-882. 